PROCEEDINGS OF THE

AMERICAN MATHEMATICAL SOCIETY

Volume 126, Number 6, June 1998, Pages 1697-1708

S 0002-9939(98)04303-2

\title{
ANGULAR DERIVATIVES AT BOUNDARY FIXED POINTS FOR SELF-MAPS OF THE DISK
}

\author{
PIETRO POGGI-CORRADINI
}

(Communicated by Theodore W. Gamelin)

\begin{abstract}
Let $\phi$ be a one-to-one analytic function of the unit disk $\mathbb{D}$ into itself, with $\phi(0)=0$. The origin is an attracting fixed point for $\phi$, if $\phi$ is not a rotation. In addition, there can be fixed points on $\partial \mathbb{D}$ where $\phi$ has a finite angular derivative. These boundary fixed points must be repelling (abbreviated b.r.f.p.). The Kœnigs function of $\phi$ is a one-to-one analytic function $\sigma$ defined on $\mathbb{D}$ such that $\phi=\sigma^{-1}(\lambda \sigma)$, where $\lambda=\phi^{\prime}(0)$. If $\phi_{K}$ is the first iterate of $\phi$ that does have b.r.f.p., we compute the Hardy number of $\sigma$, $h(\sigma)=\sup \left\{p>0: \sigma \in H^{p}(\mathbb{D})\right\}$, in terms of the smallest angular derivative of $\phi_{K}$ at its b.r.f.p.. In the case when no iterate of $\phi$ has b.r.f.p., then $\sigma \in \bigcap_{p<\infty} H^{p}$, and vice versa. This has applications to composition operators, since $\sigma$ is a formal eigenfunction of the operator $C_{\phi}(f)=f \circ \phi$. When $C_{\phi}$ acts on $H^{2}(\mathbb{D})$, by a result of C. Cowen and B. MacCluer, the spectrum of $C_{\phi}$ is determined by $\lambda$ and the essential spectral radius of $C_{\phi}, r_{e}\left(C_{\phi}\right)$. Also, by a result of P. Bourdon and J. Shapiro, and our earlier work, $r_{e}\left(C_{\phi}\right)$ can be computed in terms of $h(\sigma)$. Hence, our result implies that the spectrum of $C_{\phi}$ is determined by the derivative of $\phi$ at the fixed point $0 \in \mathbb{D}$ and the angular derivatives at b.r.f.p. of $\phi$ or some iterate of $\phi$.
\end{abstract}

\section{INTRODUCTION}

Let $\phi$ be a one-to-one and analytic function of the unit disk $\mathbb{D}$ into itself, with $\phi(0)=0$, and which is not a rotation. Then $0<\left|\phi^{\prime}(0)\right|<1$ and, by Schwarz's Lemma, the iterates of $\phi$ converge uniformly on compact subsets of $\mathbb{D}$ to 0 . A classical result of Kœnigs yields a one-to-one analytic function $\sigma$ defined on $\mathbb{D}$, with image $G=\sigma(\mathbb{D})$, such that

$$
\sigma \circ \phi=\lambda \sigma
$$

where $\lambda=\phi^{\prime}(0)$. Thus, $\lambda G \subset G$, and the action of $\phi$ on $\mathbb{D}$ is conjugated through $\sigma$ to multiplication by $\lambda$ on $G$. For this reason, the set $G$ is called a geometric model. Kønigs's theorem was introduced in the context of iteration theory to study the iterates of $\phi$ near the fixed point 0 . Here we will relate the growth of $\sigma$ near $\partial \mathbb{D}$ and the geometry of the image region $G$ to the dynamics of $\phi$ near $\partial \mathbb{D}$. We will write $\phi_{n}$ to denote the $n$th iterate of $\phi$.

Received by the editors January 18, 1996 and, in revised form, November 15, 1996.

1991 Mathematics Subject Classification. Primary 30D05, 30D55, 30C35, 47B38, 58F23.

The author was supported by the University of Washington Math. Department while at MSRI, Berkeley, in the Fall of 1995. He also wishes to thank Professor D. Marshall for his help and advice.

(C)1998 American Mathematical Society 
Note that under our assumptions, the function $\phi$ does not need to extend, even continuously, to $\partial \mathbb{D}$. However, one can consider classical generalized notions of limit and derivative at a point $\zeta \in \partial \mathbb{D}$, namely the notions of non-tangential limit and angular derivative. In this article, we will examine points $\zeta \in \partial \mathbb{D}$ where $\phi$ has non-tangential limit $\zeta$, hence can be called fixed, and where $\phi$ has a finite angular derivative, which we will call the multiplier at $\zeta$ and write as $\phi^{\prime}(\zeta)$. It follows from the Denjoy-Wolff theorem that $\phi^{\prime}(\zeta)>1$; see [9], p. 78, for a recent exposition. So, following the classification of fixed points in the theory of the iteration of rational maps, we can call such a point $\zeta$ a boundary repelling fixed point (b.r.f.p.) for $\phi$.

Recall that, for $0<p<\infty$, the Hardy space $H^{p}(\mathbb{D})$ is the space of analytic functions $f$ on $\mathbb{D}$ such that

$$
\sup _{0<r<1} \int_{0}^{\infty}\left|f\left(r e^{i \theta}\right)\right|^{p} d \theta<\infty .
$$

Our starting point is a result of [10] which states that if a map $\phi$ as above has a b.r.f.p., then the geometric model $G=\sigma(\mathbb{D})$ contains a twisted sector (see Definition 4.1 ), and $\sigma$ does not belong to $H^{p}(\mathbb{D})$ for $p$ large. In [6] and [7], we completely characterized the connection between the Hardy class of $\sigma$, i.e. the $p$ 's for which $\sigma \in H^{p}(\mathbb{D})$, and the geometry of $G=\sigma(\mathbb{D})$.

The main purpose of this paper is to relate the b.r.f.p. of $\phi$ or iterates of $\phi$ to the geometry of $G=\sigma(\mathbb{D})$ and to the "size" of the Kønigs map $\sigma$. Namely, we will find an exact formula for the Hardy number of $\sigma$ (see Definition 2.1) in terms of the multipliers at the b.r.f.p.. To do this we need to deal with a generalized notion of "basin of attraction" near each b.r.f.p..

Our study has application to the spectral theory of the composition operator $C_{\phi}$ acting on the analytic functions $f$ defined on $\mathbb{D}$ by $C_{\phi}(f)=f \circ \phi$. In fact, we will show that the shape of the spectrum for the operator $C_{\phi}$ acting on the classical Hardy space $H^{2}(\mathbb{D})$ is completely determined by $\phi^{\prime}(0)$ and the multipliers at the b.r.f.p. This result is in agreement with the work of H. Kamowitz [4], who, with entirely different techniques, analyzed the spectra of composition operators $C_{\phi}$ acting on $H^{p}(\mathbb{D}), 1 \leq p<\infty$, under the assumptions that the symbol $\phi$ is analytic in a neighborhood of $\overline{\mathbb{D}}$.

\section{Preliminaries}

We first need to recall some notations that we introduced in [6] and [7]. In [6], Lemma 3.2, we considered the set $V=\operatorname{int}\left(\bigcap_{n>0} \lambda^{n} G\right) \subset G$, which we called the invariant set of $G$. Since $V$ is open, we write $V=\bigcup_{j} V_{j}$, where $V_{j}$ are the connected components of $V$. The invariant set $V$ satisfies $\lambda V=V=\lambda^{-1} V$. Moreover, if $V_{j}$ is a component of $V$, then $\lambda V_{j}=V_{k}$ is another component of $V$. Hence, given a component $V_{j}$, its orbit $\left\{\lambda^{k} V_{j}\right\}_{-\infty}^{+\infty}$ is either wandering, i.e. $\lambda^{k} V_{j} \cap V_{j}=\emptyset$ for all $k \neq 0$, or periodic, i.e. there is a smallest integer $K \geq 1$ such that $\lambda^{K} V_{j}=V_{j}$, and we called $K$ the period of $V_{j}$.

In [7], Lemma 6.8, we showed that when $V$ has some periodic components they all have the same period. Moreover, since for each periodic component $V_{0}$ of period $K \geq 1$ multiplication by $\lambda^{K}$ is an automorphism of $V_{0}$, there is a conformal map $\psi_{0}$ of the upper half-plane $\mathbb{H}$ onto $V_{0}$ and a number $t_{0} \in(0,1)$ such that $\psi_{0}\left(t_{0} z\right)=$ $\lambda^{K} \psi_{0}(z)$ ([7], Lemma 6.8). We say that $\psi_{0}$ is the model of $V_{0}$, and $t_{0}$ is the step 
of $V_{0}$. Also we call $\Gamma_{0}=\psi_{0}(\{y i: y>0\})$ the axis of $V_{0}$. Note that $\Gamma_{0}$ is the only geodesic in the hyperbolic metric of $V_{0}$ that is invariant under multiplication by $\lambda^{K}$; in particular, $\Gamma_{0}$ connects 0 to infinity.

Definition 2.1. If $\psi$ is an analytic function on $\mathbb{D}$, we let $h(\psi)=\sup \{0<p<\infty$ : $\left.\psi \in H^{p}(\mathbb{D})\right\} \in[0, \infty]$ be the Hardy number of $\psi$.

Recall that $H^{p}(\mathbb{D}) \subset H^{p^{\prime}}(\mathbb{D})$ if $p>p^{\prime}$. So the Hardy class of $\psi$ is an interval: either $(0, h(\psi))$ or $(0, h(\psi)]$. If $\Omega$ is a simply connected set, then given any pair of one-to-one and analytic maps of $\mathbb{D}$ onto $\Omega, \psi$ and $\tilde{\psi}$, we have $\psi \in H^{p}(\mathbb{D})$ if and only if $\tilde{\psi} \in H^{p}(\mathbb{D})$. So in this case we can write that $\Omega \in H^{p}(\mathbb{D})$. Likewise, we set $h(\Omega)=\sup \left\{0<p<\infty: \Omega \in H^{p}(\mathbb{D})\right\}$.

In [7], we showed that the Hardy number of the Kœnigs map $\sigma$ can be computed in terms of the invariant set $V$ of $G$ :

$$
h(\sigma)=\min h\left(V_{j}\right)
$$

ranging over all the components $V_{j}$ of $V$. Also, we showed that for every wandering component we have $h\left(V_{j}\right)=\infty$, and that for a periodic component $V_{0}$ with period $K$ and step $t_{0}$ we have $h\left(V_{0}\right)=\log \left(t_{0}\right) / \log \left(|\lambda|^{K}\right)$. So (2.1) means that either $V$ has no periodic components in which case $h(\sigma)=\infty$, or there is a periodic component $V_{0}$ such that $h(\sigma)=h\left(V_{0}\right)=\log \left(t_{0}\right) / \log \left(|\lambda|^{K}\right)<\infty$.

Recall that a composition operator with symbol $\phi$ is defined by $C_{\phi}(f)=f \circ \phi$, and is bounded on the Hardy spaces $H^{p}(\mathbb{D}), 0<p<\infty$. In [7], together with some recent results of P. Bourdon and J. Shapiro, we obtained a formula, in terms of $h(\sigma)$, for the essential spectral radius, $r_{e}\left(C_{\phi}\right)$, of the composition operator $C_{\phi}$ acting on $H^{2}(\mathbb{D})$ :

$$
r_{e}\left(C_{\phi}\right)=|\lambda|^{h(\sigma) / 2}
$$

The case $r_{e}\left(C_{\phi}\right)=0$ corresponds to $h(\sigma)=\infty$, and therefore to the case when the invariant set $V$ does not have periodic components. When $r_{e}\left(C_{\phi}\right)>0$, then $V$ has periodic components, say of period $K \geq 1$, and there exists a periodic component $V_{0}$ with step $t_{0}$ such that $r_{e}\left(C_{\phi}\right)=|\lambda|^{h\left(V_{0}\right) / 2}=t_{0}^{K}$.

Now let $V_{0}$ be a periodic component of the invariant set $V$ of period $K \geq 1$, and let $\Gamma_{0}$ be its axis. Note that $\sigma$ cannot be constant on an arc of $\partial \mathbb{D}$, because, being one-to-one, $\sigma \in H^{p}(\mathbb{D})$ for $p \in(0,1 / 2)$ (Theorem 3.16 of $\left.[3]\right)$. Thus, $\sigma^{-1}\left(\Gamma_{0}\right)$ is a curve in $\mathbb{D}$ tending to a unique point $\zeta\left(V_{0}\right)$ on $\partial \mathbb{D}$. Hence, the correspondence $V_{0} \mapsto \zeta\left(V_{0}\right)$, from periodic components of $V$ to points of $\partial \mathbb{D}$, is well-defined. Our main theorem will show that this correspondence is injective and that its range consists of all the b.r.f.p. of $\phi_{K}$. We call $W=\sigma^{-1}(V)=\operatorname{int}\left(\bigcap_{n \geq 0} \phi_{n}(\mathbb{D})\right)$ the invariant set of $\phi$. Note that $W_{0}=\sigma^{-1}\left(V_{0}\right)$ is a connected component of $W$ such that $\phi_{K}\left(W_{0}\right)=W_{0}=\phi_{K}^{-1}\left(W_{0}\right)$, i.e. $\phi_{K}$ is an automorphism of $W_{0}$. Also, 0 and $\zeta\left(V_{0}\right)$ are in $\partial W_{0}$. The curve $\sigma^{-1}\left(\Gamma_{0}\right)$, which connects 0 to $\zeta\left(V_{0}\right)$, is the unique geodesic in the hyperbolic metric of $W_{0}$ that is fixed by $\phi_{K}$. We will show that $\sigma^{-1}\left(\Gamma_{0}\right)$ is perpendicular to $\partial \mathbb{D}$ at $\zeta\left(V_{0}\right)$, and that the set $W_{0}$ has an inner tangent at $\zeta\left(V_{0}\right)$. That is to say, for every $\beta \in(0, \pi / 2)$ there is an $\epsilon=\epsilon(\beta)>0$ such that the truncated cone

$$
\left\{z \in \mathbb{D}:\left|z-\zeta\left(V_{0}\right)\right|<\epsilon,\left|\arg \left(\zeta\left(V_{0}\right)\right)-\arg \left(\zeta\left(V_{0}\right)-z\right)\right|<\beta\right\}
$$

is contained in $W_{0}$. In particular, it follows that

$$
W_{0}=\left\{z \in \mathbb{D}: \phi_{n K}^{-1}(z) \rightarrow \zeta\left(V_{0}\right) \text { non-tangentially as } n \rightarrow \infty\right\}
$$


So, $W_{0}$ can be thought of as a "basin of attraction" for $\phi_{K}^{-1}$ at $\zeta\left(V_{0}\right)$.

Finally, by a theorem of Ostrowski (see Theorem 11.5 of [8] or Theorem 3 of $[5]), W_{0}$ has an inner tangent at $\zeta\left(V_{0}\right)$ if and only if for any Riemann map $F$ of $\mathbb{D}$ onto $W_{0}$ which fixes $\zeta\left(V_{0}\right)$, the argument of the derivative of $F$ converges to 0 non-tangentially at $\zeta\left(V_{0}\right)$. In the last section of this paper, we give a negative answer to the following natural question: does $W_{0}$ have an angular derivative at $\zeta\left(V_{0}\right)$ ? In our example, the set $W_{0}$ is actually equal to $\operatorname{int}\left(\bigcap_{n \geq 0} \phi_{n}(\mathbb{D})\right)$ and has an inner tangent at 1 . Every iterate $\phi_{n}$ has a boundary fixed point at 1 and has a finite angular derivative at 1 . So, the sets $\phi_{n}(\mathbb{D})$ all have an angular derivative at 1 with respect to $\partial \mathbb{D}$. But, $W_{0}$ does not, i.e. any Riemann map $F$ of $\mathbb{D}$ onto $W_{0}$ that fixes 1 does not have an angular derivative at 1 .

\section{Statement of the main Result}

We establish a one-to-one correspondence between the periodic components of $V$, and the b.r.f.p. of the iterate $\phi_{K}$, where $K \geq 1$ is the common period of the periodic components. Also, we obtain a formula connecting the multiplier at a given b.r.f.p. and the Hardy number of the corresponding periodic component. This yields the following dichotomy. Either no iterate of $\phi$ has a finite angular derivative at a boundary fixed point - and this happens if and only if the invariant set $V$ has no periodic components, hence if and only if $h(\sigma)=\infty$ - or else the iterates of $\phi_{K}$, where $K$ is the common period of the periodic components of $V$, are the only iterates of $\phi$ that do have b.r.f.p. Moreover, in the latter case, in view of the result of [7] mentioned above, we obtain a formula for $h(\sigma)$ in terms of the smallest angular derivative of $\phi_{K}$ at its boundary fixed points.

Theorem 3.1. Let $\phi: \mathbb{D} \rightarrow \phi(\mathbb{D}) \subset \mathbb{D}$ be analytic and one-to-one, with $\phi(0)=0$, and $\phi$ not a rotation. Let $\sigma$ be the Konigs function of $\phi, G=\sigma(\mathbb{D}), \lambda=\phi^{\prime}(0)$, and $V=\operatorname{int}\left(\bigcap_{n \geq 0} \lambda^{n} G\right)$. Assume that $V$ has at least one periodic component. Let $K$ denote the common period of the periodic components of $V$. For every periodic component $V_{j}$ of $V$, let $\psi_{j}, t_{j}$ and $\Gamma_{j}$ denote respectively the model, the step and the axis of $V_{j}$. Finally, let $\zeta\left(V_{j}\right)=\overline{\sigma^{-1}\left(\Gamma_{j}\right)} \cap \partial \mathbb{D}$. Then:

(i) $\zeta\left(V_{0}\right)=\zeta\left(V_{1}\right)$ implies $V_{0}=V_{1}$.

(ii) A point $\zeta \in \partial \mathbb{D}$ is a boundary fixed point of $\phi_{n}, n \geq 1$, and $\phi_{n}$ has a finite angular derivative at $\zeta$ if and only if $\zeta=\zeta\left(V_{0}\right)$ for some periodic component $V_{0}$ of $V$ and $n=m K$ for some $m=1,2, \ldots$.

(iii) If $A>1$ is the angular derivative of $\phi_{K}$ at $\zeta\left(V_{0}\right)$, and $t_{0}$ is the step of $V_{0}$, then $A=1 / t_{0}$.

(iv) The curve $\sigma^{-1}\left(\Gamma_{0}\right)$ is perpendicular to $\partial \mathbb{D}$ at $\zeta\left(V_{0}\right)$, and $W_{0}=\sigma^{-1}\left(V_{0}\right)$ has an inner tangent at $\zeta\left(V_{0}\right)$.

The next corollary follows from Theorem 3.1 (ii).

Corollary 3.2. Let $\phi: \mathbb{D} \rightarrow \phi(\mathbb{D}) \subset \mathbb{D}$ be analytic and one-to-one, with $\phi(0)=0$, and $\phi$ not a rotation. Then, one of the following holds:

(I) No iterate $\phi_{n}, n=1,2 \ldots$, has a finite angular derivative at a boundary fixed point.

(II) There is an integer $K \geq 1$ such that $\phi_{n}$ has a finite angular derivative at a boundary fixed point if and only if $n=m K$ for some $m=1,2, \ldots$. 
Definition 3.3. We say that $\phi$ is of type (I) when $\phi$ is as in Corollary 3.2 (I), and of type (II) otherwise. If $\phi$ is of type (II), we set

$$
A(\phi)=\min \left\{\phi_{K}^{\prime}(\zeta): \zeta \in \partial \mathbb{D}, \phi_{K}(\zeta)=\zeta\right\}>1
$$

where $K$ is given by Corollary $3.2(\mathrm{II})$.

Note that the minimum in (3.1) is attained because it is attained in (2.1) and because of the one-to-one correspondence of Theorem 3.1 between periodic components and b.r.f.p. (see also Proposition 2.46 of [2]).

Corollary 3.4. Let $\phi: \mathbb{D} \rightarrow \phi(\mathbb{D}) \subset \mathbb{D}$ be analytic and one-to-one, with $\phi(0)=0$, and $\phi$ not a rotation. Let $\lambda=\phi^{\prime}(0)$ and let $\sigma$ be the Konigs map of $\phi$. Put $h(\sigma)=\sup \left\{p>0: \sigma \in H^{p}(\mathbb{D})\right\}$.

Then $h(\sigma)=\infty$ if $\phi$ is of type (I), while if $\phi$ is of type (II), then

$$
h(\sigma)=\frac{\log A(\phi)}{\log \left(1 /|\lambda|^{K}\right)} .
$$

Proof. If $\phi$ is of type (I), $V$ has no periodic components, by Theorem 3.1 (ii); hence $h(\sigma)=\infty$ by (2.1). Otherwise, the formula for $h(\sigma)$ follows from Theorem 3.1 (iii) by the remarks right after (2.1).

Remark 3.5. Since $\sigma$ is univalent, $h(\sigma) \geq 1 / 2$ (see Theorem 3.16 of [3]). So Corollary 3.4 implies that $A(\phi) \geq|\lambda|^{-K / 2}>1$.

Finally, C. Cowen and B. MacCluer (see [1] or Theorem 7.30 on p. 289 of [2]) showed that, under the same assumptions on $\phi$ as in Theorem 3.1, the spectrum of $C_{\phi}$ acting on $H^{2}(\mathbb{D})$ has the following form:

$$
\left\{z \in \mathbb{C}:|z| \leq r_{e}\left(C_{\phi}\right)\right\} \cup\left\{\phi^{\prime}(0)^{n}: n=1,2, \ldots\right\} \cup\{1\} .
$$

Our next result allows one to determine the shape of the spectrum of $C_{\phi}$ in terms of the derivative of the symbol $\phi$ at the origin and the possible angular derivatives at boundary fixed points of $\phi$ or some iterate of $\phi$.

Corollary 3.6. Let $\phi: \mathbb{D} \rightarrow \phi(\mathbb{D}) \subset \mathbb{D}$ be analytic and one-to-one, with $\phi(0)=0$, and $\phi$ not a rotation. Let $C_{\phi}(f)=f \circ \phi$ be the composition operator with symbol $\phi$ acting on $H^{2}(\mathbb{D})$, and let $r_{e}\left(C_{\phi}\right)$ be the essential spectral radius of $C_{\phi}$.

Then $r_{e}\left(C_{\phi}\right)=0$ if $\phi$ is of type (I), while if $\phi$ is of type (II), then

$$
r_{e}\left(C_{\phi}\right)=\left(\frac{1}{A(\phi)}\right)^{\frac{1}{2 K}}
$$

Proof. Use (2.2) and Corollary 3.4.

\section{Proof of the main theorem}

We start by showing the injectivity, part $(i)$ of Theorem 3.1. Consider two periodic components $V_{0}, V_{1}$ with $\zeta\left(V_{0}\right)=\zeta\left(V_{1}\right)=\zeta$, and assume that $V_{0}$ and $V_{1}$ are distinct. For $j=0,1$, let $W_{j}=\sigma^{-1}\left(V_{j}\right)$, let $\Gamma_{j}$ be the axis of $V_{j}$ and let $\gamma_{j}=\sigma^{-1}\left(\Gamma_{j}\right)$. As we said above, $\gamma_{0}$ and $\gamma_{1}$ are curves in $\mathbb{D}$ connecting 0 to $\zeta$. So they bound a simply connected region $\Omega \subset \mathbb{D}$ such that $\partial \Omega=\{0\} \cup \gamma_{0} \cup \gamma_{1} \bigcup\{\zeta\}$, and in particular $\bar{\Omega} \cap \partial \mathbb{D}=\{\zeta\}$. Recall that $V_{0}$ and $V_{1}$ have the same period $K \geq 1$. Hence, $\phi_{K}\left(\gamma_{j}\right)=\gamma_{j}$, for $j=0,1$. Also, $\phi_{K}(\Omega)$ is a simply connected region in $\mathbb{D} \backslash\left(\gamma_{0} \cup \gamma_{1}\right)$ whose boundary coincide with $\partial \Omega$. Therefore, $\phi_{K}(\Omega)=\Omega$. So $\Omega$ is contained in the invariant set of $\phi$, i.e. $\Omega \subset W=\operatorname{int}\left(\bigcap_{n \geq 0} \phi_{n}(\mathbb{D})\right)$. On the other 
hand, $\Omega \cap W_{j} \neq \emptyset$ for $j=0,1$, which is a contradiction since $W_{0}$ and $W_{1}$ are distinct connected components of $W$. Thus, $(i)$ is proved.

Now we prove part $(i i)$ of Theorem 3.1. First, assume that $V_{0}$ is a periodic component. Note that $\phi_{m K}(z)$ tends to $\zeta\left(V_{0}\right)$ as $z$ tends to $\zeta\left(V_{0}\right)$ along $\sigma^{-1}\left(\Gamma_{0}\right)$. So, by Lindelöf, $\phi_{m K}$ has non-tangential limit $\zeta\left(V_{0}\right)$ at $\zeta\left(V_{0}\right)$. Moreover, for $w \in$ $\sigma^{-1}\left(\Gamma_{0}\right)$, if $\rho_{\mathbb{D}}$ and $\rho_{\mathbb{H}}$ denote respectively the hyperbolic distance on $\mathbb{D}$ and on $\mathbb{H}$,

$$
\rho_{\mathbb{D}}\left(w, \phi_{m K}(w)\right) \leq \rho_{\mathbb{H}}\left(\psi_{0}^{-1} \circ \sigma(w), t_{0}^{m}\left(\psi_{0}^{-1} \circ \sigma(w)\right)\right)=\log \left(1 / t_{0}^{m}\right)<\infty
$$

So, by the Julia-Carathéodory theorem, $\phi_{m K}$ has a finite angular derivative at $\zeta\left(V_{0}\right)$.

For the converse we introduce the notion of a twisted sector, which first appeared in [10]. Our definition of twisted sector is phrased differently, but is equivalent, to the one in [10]. For $z \in \mathbb{C}$ and $r>0, B(z, r)=\{w \in \mathbb{C}:|z-w|<r\}$. If $E \subset \mathbb{C} \backslash\{0\}$, and $\epsilon>0$, we set

$$
S_{\epsilon}[E]=\bigcup_{z \in E} B(z, \epsilon|z|) .
$$

Definition 4.1. Let $\gamma$ be a closed and connected subset of $\mathbb{C} \backslash\{0\}$ such that 0 and $\infty$ belong to the closure of $\gamma$ in the Riemann sphere. Let $\epsilon>0$. We say that $S_{\epsilon}[\gamma]$ is a twisted sector of width $\epsilon$ supported on $\gamma$.

Remark 4.2. Note that if $S_{\epsilon}[\gamma]$ is a twisted sector of width $\epsilon>0$ supported on $\gamma$, then for every $c \in \mathbb{C} \backslash\{0\}, c S_{\epsilon}[\gamma]$ is also a twisted sector of width $\epsilon$ supported on $c \gamma$, since $c S_{\epsilon}[\gamma]=S_{\epsilon}[c \gamma]$.

Assume that $\zeta_{0} \in \partial \mathbb{D}$ is a b.r.f.p. of $\phi_{n}, n \geq 1$. We need to show that the set $V=\operatorname{int}\left(\bigcap_{j \geq 0} \lambda^{j} G\right)$ does have periodic components, say of period $K \geq 1$, that there is one periodic component $V_{0}$ such that $\zeta\left(V_{0}\right)=\zeta_{0}$, and that $n=m K$ for some integer $m \geq 1$. Suppose that we can find a component $V_{0}$ of the set $V^{(n)}=\operatorname{int}\left(\bigcap_{j \geq 0} \lambda^{j n} G\right)$ (the invariant set for $\phi_{n}$ ) such that $V_{0}$ is of period one with respect to multiplication by $\lambda^{n}$, i.e. $\lambda^{n} V_{0}=V_{0}$, and such that $\zeta\left(V_{0}\right)=\zeta_{0}$. Then, since $V=V^{(n)}, V_{0}$ must be a periodic component of $V$, and therefore we must have $n=m K$, where $K$ is the period of the periodic components of $V$. So we only need to show that if $\zeta \in \partial \mathbb{D}$ is a b.r.f.p. for a map $\phi$ as in Theorem 3.1, then the corresponding invariant set $V$ has a periodic component $V_{0}$ of period 1 such that $\zeta\left(V_{0}\right)=\zeta$.

Let $\zeta \in \partial \mathbb{D}$ be fixed by $\phi$ and assume that $\phi$ has a finite angular derivative $A>1$ at $\zeta$. For convenience, let's move to the upper-half plane $\mathbb{H}$ via the map $\tau(z)=\zeta(z-i) /(z+i)$; hence let $\Phi=\tau^{-1} \circ \phi \circ \tau$ and $\Sigma=\sigma \circ \tau$. Then, infinity is a boundary fixed point for $\Phi$ and $\Phi$ has a finite angular derivative there, i.e. $z / \Phi(z)$ converges to a number $A>1$ as $z$ tends to infinity non-tangentially. Let $\gamma=\Sigma(\{y i: y \geq 1\})$. Then, in the proof of Proposition 3.3 of [10] it is shown that $\gamma$ supports a twisted sector of width $\epsilon$ that is contained in $G$, for some $\epsilon>0$. For all $n \in \mathbb{N}$, let $\gamma_{n}=\lambda^{n} \gamma$. Recall that, by Schwarz's lemma, $0<|\lambda|=\left|\phi^{\prime}(0)\right|<1$. By Remark 4.2

$$
S_{\epsilon}\left[\gamma_{n}\right]=\lambda^{n} S_{\epsilon}[\gamma] \subset \lambda^{n} G .
$$

Since $\gamma_{n}$ connects 0 to $\infty$, for every $n \in \mathbb{N}$ we can find $\zeta_{n} \in \gamma_{n} \cap \partial \mathbb{D}$. Also, $\gamma_{n}$ is parameterized by $\Sigma \circ \Phi_{n}(y i)$, for $y \geq 1$, so we can assume that $\zeta_{n}=\Sigma \circ \Phi_{n}\left(y_{n} i\right)$ is the last point of $\gamma_{n}$ belonging to $\overline{\mathbb{D}}$. Set $\tilde{\gamma}_{n}=\Sigma \circ \Phi_{n}\left(\left\{y i: y \geq y_{n}\right\}\right)$. 
Claim 4.3. There is a connected component $V_{0}$ of the invariant set $V$ of $G$, such that for every integer $N_{0}<\infty$ there is $N>N_{0}$ with $S_{\epsilon / 2}\left[\tilde{\gamma}_{N}\right] \backslash \mathbb{D} \subset V_{0}$.

Proof. By (4.1), $B\left(\zeta_{n}, \epsilon\right) \subset \lambda^{n} G$. Let $\zeta_{n_{k}}$ be a subsequence converging to $\zeta \in \partial \mathbb{D}$; then for some $k_{0} \in \mathbb{N}$, and for every $k \geq k_{0}$, we have

$$
B(\zeta, \epsilon / 2) \subset B\left(\zeta_{n_{k}}, \epsilon\right) \subset \lambda^{n_{k}} G
$$

Hence $B(\zeta, \epsilon / 2) \subset \bigcap_{n \geq 0} \lambda^{n} G$, i.e. $B(\zeta, \epsilon / 2) \subset V$. Let $V_{0}$ be the connected component of $V$ containing $B(\zeta, \epsilon / 2)$. We can assume that $\tilde{\gamma}_{n_{k}} \cap V_{0} \neq \emptyset$, for all $k \in \mathbb{N}$. Now, suppose that for infinitely many $k$ we can find $z_{k} \in\left(S_{\epsilon / 2}\left[\tilde{\gamma}_{n_{k}}\right] \backslash \mathbb{D}\right) \cap \partial V_{0}$. Since $z_{k} \in S_{\epsilon / 2}\left[\tilde{\gamma}_{n_{k}}\right]$, there is $z \in \tilde{\gamma}_{n_{k}}$ such that $\left|z_{k}-z\right|<(\epsilon / 2)|z|$; in particular $\left|z_{k}\right|<(1+\epsilon / 2)|z|$, so by $(4.1)$

$$
B\left(z_{k}, c\left|z_{k}\right|\right) \subset B(z, \epsilon|z|) \subset \lambda^{n_{k}} G
$$

where $c=(\epsilon / 2) /(1+\epsilon / 2)$. Now, multiply each $z_{k}$ by $\lambda^{m_{k}}$, where the power $m_{k} \in \mathbb{N}$ is chosen so that for all $k \in \mathbb{N}$

$$
\tilde{z}_{k}=\lambda^{m_{k}} z_{k} \in Q=\left\{z \in \mathbb{C}: 1 \leq|z| \leq|\lambda|^{-1}\right\} .
$$

Notice that $m_{k} \geq 0$, because $z_{k} \notin \mathbb{D}$. Then, for infinitely many $k$ we have:

$$
\text { (a) } B\left(\tilde{z}_{k}, c\left|\tilde{z}_{k}\right|\right) \subset \lambda^{n_{k}} G \quad \text { and } \quad(b) \tilde{z}_{k} \in \partial\left(\lambda^{m_{k}} V_{0}\right) \text {. }
$$

Again assume, by passing to a subsequence, that $\tilde{z}_{k}$ converges to a point $\xi \in Q$. By the same argument as above, using $(a), B(\xi, c|\xi|)$ is contained in the invariant set $V$. Let $V_{1}$ be the connected component of $V$ containing $B(\xi, c|\xi|)$. Choose $\tilde{z}_{k} \in B(\xi, c|\xi|)$. Then, by $(b), V_{1} \cap \lambda^{m_{k}} V_{0} \neq \emptyset$. Hence $V_{1}=\lambda^{m_{k}} V_{0}$ and $\tilde{z}_{k} \in \lambda^{m_{k}} V_{0}$, which contradicts $(b)$. Therefore, there is $k_{0} \in \mathbb{N}$ such that, for $k \geq k_{0}$,

$$
\left(S_{\epsilon / 2}\left[\tilde{\gamma}_{n_{k}}\right] \backslash \mathbb{D}\right) \subset V_{0} .
$$

From the proof of Claim 4.3, we could already deduce that $V_{0}$ must be a periodic component of $V$. But, we also want to show that its period must be 1 . That's the content of our next claim

Claim 4.4. The component $V_{0}$ is periodic of period 1, i.e. $\lambda V_{0}=V_{0}=\lambda^{-1} V_{0}$.

Proof. Let $\tilde{\gamma}=\tilde{\gamma}_{N}$ be given as in Claim 4.3.

Definition 4.5. Let $\Omega$ be a simply connected region with hyperbolic metric $\rho_{\Omega}$, and let $F \subset \Omega$. We say that $U$ is a hyperbolic neighborhood of $F$ in $\Omega$, if

$$
U=\bigcup_{z \in F}\left\{w \in \Omega: \rho_{\Omega}(w, z)<C\right\}
$$

for some constant $0<C<\infty$.

Then, $S_{\epsilon}[\tilde{\gamma}]$ contains a hyperbolic neighborhood of $\tilde{\gamma}$ in $G$. In fact, if $w \in G$ satisfies $\rho_{G}(w, z)<C$, for some $z \in \tilde{\gamma}$, then

$$
\frac{1}{2} \log \left(1+\frac{|w-z|}{d(z, \partial G)}\right)<C .
$$

See [10], Lemma 3.2. But, since $G \neq \mathbb{C}$, there is a constant $C_{1}>0$ such that $d(z, \partial G) \leq C_{1}|z|$. Hence, if $C$ is chosen small enough,

$$
|w-z| \leq C_{1}\left(e^{2 C}-1\right)|z| \leq(\epsilon / 2)|z| .
$$


On the other hand, notice that $\Sigma^{-1}(\tilde{\gamma})=\Phi_{N}\left(\left\{y i: y \geq y_{N}\right\}\right)$. The fact that $\Phi$ has an angular derivative at infinity implies that $\Phi_{N}$ also has an angular derivative at infinity. So $\Sigma^{-1}(\tilde{\gamma})$ is asymptotic in the Riemann sphere to the imaginary axis at infinity, i.e. given any hyperbolic neighborhood of $\Sigma^{-1}(\tilde{\gamma})$ in $\mathbb{H}$, the upper half imaginary axis is eventually contained in it. So, for $R>0$ large enough, $\Sigma(\{y i: y \geq R\})$ is contained in $S_{\epsilon / 2}[\tilde{\gamma}]$. By the same argument, we also have $R^{\prime}>R>0$, such that $\Sigma \circ \Phi\left(\left\{y i: y \geq R^{\prime}\right\}\right)$ is contained in $S_{\epsilon / 2}[\tilde{\gamma}]$. That is to say, $\Sigma\left(\left\{y i: y \geq R^{\prime}\right\}\right)$ and $\lambda \Sigma\left(\left\{y i: y \geq R^{\prime}\right\}\right)$ are both contained in $S_{\epsilon / 2}[\tilde{\gamma}]$. But, since $S_{\epsilon / 2}[\tilde{\gamma}] \backslash \mathbb{D} \subset V_{0}$, we must have $\lambda V_{0}=V_{0}$.

Let $\Gamma_{0}$ be the axis of $V_{0}$. The preimage $\Sigma^{-1}\left(\Gamma_{0}\right)$ is a curve in $\mathbb{H}$ starting at $i$ and converging to a unique point of $\partial \mathbb{H}$ in the Riemann sphere. The next claim shows that such a point must be infinity, and hence proves the converse, completing the proof of part (ii) of Theorem 3.1.

Claim 4.6. The curve $\Sigma^{-1}\left(\Gamma_{0}\right)$ is contained in a hyperbolic neighborhood of $\{y i$ : $y \geq 1\}$ in $\mathbb{H}$.

Proof. Fix $0<\delta<\pi / 2$, and consider

$$
U_{\delta}=\{z \in \mathbb{H}:|\arg (z)-\pi / 2|<\pi / 2-\delta\} .
$$

Let $K_{\delta}=\mathbb{H} \backslash U_{\delta}$. We claim that we can choose $\delta$ small enough so that for every $z \in \psi_{0}\left(K_{\delta}\right), d\left(z, \partial V_{0}\right)<(\epsilon / 4)|z|$. Suppose not. Then we can pick a sequence $z_{n} \in \psi_{0}\left(K_{\delta_{n}}\right)$, with $\delta_{n}$ tending to zero as $n$ tends to infinity, such that for all $n \in \mathbb{N}$

$$
d\left(z_{n}, \partial V_{0}\right) \geq \frac{\epsilon}{4}\left|z_{n}\right|
$$

Let $\tilde{z}_{n}=\lambda^{m_{n}} z_{n}$, with the appropriate power $m_{n}$ so that

$$
1 \leq\left|\tilde{z}_{n}\right| \leq|\lambda|^{-1}
$$

Then (4.2) still holds with $z_{n}$ replaced by $\tilde{z}_{n}$. Now, let $\xi$ be the limit of a subsequence $\tilde{z}_{n_{j}}$. For some $j_{0} \in \mathbb{N}$, and for all $j \geq j_{0}$

$$
B(\xi,(\epsilon / 8)|\xi|) \subset B\left(\tilde{z}_{n_{j}},(\epsilon / 4)\left|\tilde{z}_{n_{j}}\right|\right) \subset V_{0} .
$$

Let $\Delta$ be a small open neighborhood of $\psi_{0}^{-1}(\xi)$ in $\mathbb{H}$. Since $\delta_{n}$ tends to zero, eventually

$$
\psi_{0}^{-1}\left(\tilde{z}_{n_{j}}\right)=t_{0}^{m_{n_{j}}} \psi_{0}^{-1}\left(z_{n_{j}}\right) \notin \Delta,
$$

which is a contradiction, because eventually $\tilde{z}_{n_{j}} \in \psi_{0}(\Delta)$. Therefore, by Claim 4.3, if $\tilde{\gamma}=\tilde{\gamma}_{N}$, we can choose $\delta_{0}>0$ such that $\tilde{\gamma} \subset \psi_{0}\left(U_{\delta_{0}}\right)$, i.e. $\psi_{0}^{-1}(\tilde{\gamma}) \subset U_{\delta_{0}}$. Notice that $\psi_{0}^{-1}(\tilde{\gamma})$ is a curve which tends to infinity. Thus, $\{y i: y \geq R\}$ is contained in a hyperbolic neighborhood of $\psi_{0}^{-1}(\tilde{\gamma})$ in $\mathbb{H}$ for some $R>0$. That is to say, applying the map $\Sigma^{-1} \circ \psi_{0}, \Sigma^{-1}\left(\Gamma_{0}\right)$ is contained in a hyperbolic neighborhood of $\Sigma^{-1}(\tilde{\gamma})=\Phi_{N}\left(\left\{y i: y \geq y_{N}\right\}\right)$ in $\Sigma^{-1}\left(V_{0}\right)$. But, since the hyperbolic metric of $\mathbb{H}$ is bounded above by the hyperbolic metric of $\Sigma^{-1}\left(V_{0}\right)$, and since $\Phi_{N}\left(\left\{y i: y \geq y_{N}\right\}\right)$ is asymptotic in the Riemann sphere to the imaginary axis at infinity, we have that $\Sigma^{-1}\left(\Gamma_{0}\right)$ is contained in a hyperbolic neighborhood of $\left\{y i: y \geq R^{\prime}\right\}$, for some large $R^{\prime}$. The claim follows from this.

Now, we want to show part (iii) of Theorem 3.1. For all $n \in \mathbb{N}$, let $z_{n}=$ $\psi_{0}\left(t_{0}^{-n} i\right) \in \Gamma_{0}$, and $w_{n}=\Sigma^{-1}\left(z_{n}\right)$. We need the following geometric property of $\Sigma^{-1}\left(\Gamma_{0}\right)$. For all $n \in \mathbb{N}$, consider the curve $\tilde{\Gamma}_{n}=\Sigma^{-1} \circ \psi_{0}\left(\left\{y i: y \geq t_{0}^{-n}\right\}\right)$, which is 
the part of the curve $\Sigma^{-1}\left(\Gamma_{0}\right)$ which starts at $w_{n}$ and, by Claim 4.6 , tends to infinity in a hyperbolic neighborhood $U_{\theta}=\{z \in \mathbb{H}:|\arg (z)-\pi / 2|<\theta\}, \theta \in(0, \pi / 2)$, of the imaginary axis. Let $\tilde{w}_{n}$ be a point of $\tilde{\Gamma}_{n}$ such that

$$
\operatorname{Im}\left(\tilde{w}_{n}\right)=\min \left\{\operatorname{Im}(w): w \in \tilde{\Gamma}_{n}\right\} .
$$

Claim 4.7. There is a constant $C_{0}>0$ and some $n_{0} \in \mathbb{N}$ such that for all $n \geq n_{0}$

$$
\operatorname{Im}\left(\tilde{w}_{n}\right) \geq C_{0} \operatorname{Im}\left(w_{n}\right)
$$

Proof. Since $\Phi$ has a finite angular derivative $A>1$ at infinity, there is a constant $R>0$ such that, for all $z \in U_{\theta} \backslash\{|z| \leq R\}$,

$$
\operatorname{Im}(z) \geq \operatorname{Im}(\phi(z)) .
$$

Choose $n_{0}$ so that $\left|w_{n}\right|>R$ for all $n \geq n_{0}$. For $m \in \mathbb{N}, m \geq 1$, let $v_{m}$ be a point such that

$$
\operatorname{Im}\left(v_{m}\right)=\min \left\{\operatorname{Im}(w): \psi_{0}^{-1} \circ \Sigma(w) \in\left\{y i: t_{0}^{-(n+m)} \leq y \leq t_{0}^{-(n+m+1)}\right\}\right\} .
$$

Then

$$
\rho_{\mathbb{H}}\left(v_{m}, w_{n+m+1}\right) \leq \rho_{\mathbb{H}}\left(\psi_{0}^{-1} \circ \Sigma\left(v_{m}\right), t_{0}^{-(n+m+1)} i\right) \leq \log \left(1 / t_{0}\right) .
$$

On the other hand,

$$
\rho_{\mathbb{H}}\left(v_{m}, w_{n+m+1}\right) \geq \frac{1}{2} \log \left(1+\frac{\left|w_{n+m+1}-v_{m}\right|}{\operatorname{Im}\left(v_{m}\right)}\right) .
$$

So, for some constant $C_{0}>0$ depending only on $t_{0}$, and by $(4.3), \operatorname{Im}\left(v_{m}\right) \geq$ $C_{0} \operatorname{Im}\left(w_{n+m+1}\right) \geq C_{0} \operatorname{Im}\left(w_{n}\right)$. The claim follows because $m$ is arbitrary.

Now, consider the following sequence of maps:

$$
\Psi_{n}(w)=\lambda^{n} \Sigma\left(\operatorname{Re}\left(w_{n}\right)+w \operatorname{Im}\left(w_{n}\right)\right) .
$$

For all $n \in \mathbb{N}, \Psi_{n}$ maps $\mathbb{H}$ conformally onto $\lambda^{n} G$, and $\Psi_{n}(i)=\lambda^{n} z_{n}=z_{0}$. In particular, $\left\{\Psi_{n}\right\}$ is a normal family. Let $\Psi$ be the normal limit of a subsequence $\Psi_{n_{k}}$. Then, since $\Psi_{n_{k}}(\mathbb{H})=\lambda^{n_{k}} G, \Psi(\mathbb{H}) \subset V$, and since $\Psi_{n_{k}}(i)=z_{0}, \Psi(\mathbb{H}) \subset V_{0}$. On the other hand, $V_{0} \subset \Psi_{n_{k}}(\mathbb{H})$, for all $k \in \mathbb{N}$. So $\Psi(\mathbb{H})=V_{0}$. Moreover, by the Hurwitz theorem, $\Psi$ is one-to-one. Thus $\Psi^{-1} \circ \psi_{0}$ is an automorphism of $\mathbb{H}$ that fixes $i$.

Claim 4.8. $\Psi^{-1} \circ \psi_{0}$ is the identity map on $\mathbb{H}$.

Proof. Note that $\Psi_{n_{k}}^{-1}$ converges uniformly on compact subsets of $V_{0}$ to $\Psi^{-1}$. So, fix $m \geq 1$, and consider the compact set $K_{m}=\psi_{0}\left(\left\{y i: 1 \leq y \leq t_{0}^{-m}\right\}\right)$ in $V_{0}$. Then

$$
\Psi_{n_{k}}^{-1}\left(K_{m}\right)=\frac{\Sigma^{-1}\left(\lambda^{-n_{k}} K_{m}\right)-\operatorname{Re}\left(w_{n_{k}}\right)}{\operatorname{Im}\left(w_{n_{k}}\right)} .
$$

Since $\Sigma^{-1}\left(\lambda^{-n_{k}} K_{m}\right) \subset \Sigma^{-1}\left(\Gamma_{0}\right)$, by Claim 4.6 there is an absolute constant $0<$ $\theta<\pi / 2$ such that $\Sigma^{-1}\left(\lambda^{-n_{k}} K_{m}\right)$ is contained in a sector $U_{\theta}$. In particular, there is a constant $C_{1}>0$ such that $\left|\operatorname{Re}\left(w_{n_{k}}\right)\right| / \operatorname{Im}\left(w_{n_{k}}\right) \leq C_{1}$, for all $k$. Therefore, by (4.4), $\Psi_{n_{k}}^{-1}\left(K_{m}\right)$ is contained in $\bigcup_{-C_{1}<x<C_{1}}\left(x+U_{\theta}\right)$. Moreover, by Claim 4.7,

$$
\min \left\{\operatorname{Im}(w): w \in \Psi_{n_{k}}^{-1}\left(K_{m}\right)\right\} \geq C_{0}>0 .
$$

So, we can find a hyperbolic neighborhood $U$ of $\{y i: y \geq 1\}$ in $\mathbb{H}$ such that $\Psi_{n_{k}}^{-1}\left(K_{m}\right) \subset U$ for all $k \in \mathbb{N}$ and all $m \geq 1$. Letting $k$ tend to infinity, $\Psi_{n_{k}}^{-1}$ converges 
uniformly to $\Psi^{-1} \circ \psi_{0}$ on $\left\{y i: 1 \leq y \leq t_{0}^{-m}\right\}$. But, $\Psi^{-1} \circ \psi_{0}\left(\left\{y i: 1 \leq y \leq t_{0}^{-m}\right\}\right)$ is an arc of a geodesic in $\mathbb{H}$, since $\Psi^{-1} \circ \psi_{0}$ is an automorphism. Letting $m$ become large, we see that this geodesic must tend to $\partial \mathbb{H}$ while staying in $U$, and therefore it can only be $\{y i: y \geq 1\}$. Hence $\Psi^{-1} \circ \psi_{0}$ fixes the upper half of the imaginary axis and fixes $i$. So, it must be the identity.

We conclude that the only possible limit for any subsequence of $\left\{\Psi_{n}\right\}$ is $\psi_{0}$. Therefore, the whole sequence $\left\{\Psi_{n}\right\}$ converges to $\psi_{0}$.

We can now compute the angular derivative of $\Phi$ at infinity in terms of $V_{0}$. Let $\pi_{n}(w)=\operatorname{Re}\left(w_{n}\right)+w \operatorname{Im}\left(w_{n}\right)$. Then, $\Psi_{n}=\lambda^{n} \Sigma \circ \pi_{n}$. So,

$$
\pi_{n} \circ\left(\Psi_{n}^{-1} \circ \lambda \Psi_{n}\right)=\Phi \circ \pi_{n} .
$$

Therefore

$$
\Phi^{\prime} \circ \pi_{n}=\left(\Psi_{n}^{-1} \circ \lambda \Psi_{n}\right)^{\prime} \rightarrow\left(\psi_{0}^{-1} \circ \lambda \psi_{0}\right)^{\prime}=t_{0}
$$

as $n$ tends to infinity. In particular, $\Phi^{\prime} \circ \pi_{n}(i)=\Phi^{\prime}\left(w_{n}\right)$ tends to $t_{0}$ as $n$ tends to infinity. So, by Claim 4.6, the angular derivative of $\Phi$ at infinity is $1 / t_{0}$, completing the proof of (iii).

Finally, we show part $(i v)$ of Theorem 3.1. Note that $\sigma^{-1}\left(\Gamma_{0}\right)$ is perpendicular to $\partial \mathbb{D}$ at $\zeta\left(V_{0}\right)$ if and only if $\sigma^{-1}\left(\Gamma_{0}\right)$ is eventually contained in any hyperbolic neighborhood of the ray $\left[0, \zeta\left(V_{0}\right)\right]$ in $\mathbb{D}$. So, consider $I_{1}=\left\{y i: 1 \leq y \leq t_{0}^{-1}\right\}$. Then, $\Psi_{n}^{-1} \circ \psi_{0}$ converges to the identity map on $I_{1}$. So, $\Psi_{n}^{-1} \circ \psi_{0}\left(I_{1}\right)$ is eventually contained in any hyperbolic neighborhood of $I_{1}$. Notice that

$$
\Sigma^{-1}\left(\Gamma_{0}\right)=\bigcup_{k=-\infty}^{+\infty} \pi_{k} \circ \Psi_{k}^{-1} \circ \psi_{0}\left(I_{1}\right)
$$

Therefore, if $P=\bigcup_{k=-\infty}^{+\infty} \pi_{k}\left(I_{1}\right)$, then $\Sigma^{-1}\left(\Gamma_{0}\right)$ is eventually contained in any hyperbolic neighborhood of $P$. Note that $\pi_{k}\left(I_{1}\right)=\left[w_{n}, w_{n}+i\left(t_{0}^{-1}-1\right) \operatorname{Im}\left(w_{n}\right)\right]$. So, to show that $\Sigma^{-1}\left(\Gamma_{0}\right)$ is eventually contained in any hyperbolic neighborhood of the imaginary axis, it is enough to show this for the sequence $\left\{w_{n}\right\}_{n=0}^{\infty}$. Notice that

$$
\frac{\operatorname{Re}\left(w_{n+1}\right)-\operatorname{Re}\left(w_{n}\right)}{\operatorname{Im}\left(w_{n}\right)}+i \frac{\operatorname{Im}\left(w_{n+1}\right)}{\operatorname{Im}\left(w_{n}\right)}=\Psi_{n}^{-1} \circ \psi_{0}\left(t_{0}^{-1} i\right) \longrightarrow t_{0}^{-1} i
$$

as $n$ tends to infinity. So,

$$
\frac{\left|\operatorname{Re}\left(w_{n+1}\right)-\operatorname{Re}\left(w_{n}\right)\right|}{\operatorname{Im}\left(w_{n+1}\right)-\operatorname{Im}\left(w_{n}\right)}=\frac{\left|\operatorname{Re}\left(w_{n+1}\right)-\operatorname{Re}\left(w_{n}\right)\right|}{\operatorname{Im}\left(w_{n}\right)} \frac{1}{\frac{\operatorname{Im}\left(w_{n+1}\right)}{\operatorname{Im}\left(w_{n}\right)}-1} \longrightarrow 0
$$

as $n$ tends to infinity, by (4.5).

To show that $\sigma^{-1} \circ \psi_{0}(\mathbb{H})$ has an inner tangent at $\zeta\left(V_{0}\right)$ we proceed similarly, by considering the fact that $\Psi_{n}^{-1} \circ \psi_{0}$ converges to the identity map on every fixed hyperbolic neighborhood of $I_{1}$. So Theorem 3.1 is proved.

\section{REMARK ON THE DEGREE OF CONTACT OF PERIODIC COMPONENTS}

Does the map $\Sigma^{-1} \circ \psi_{0}$ have an angular derivative at infinity? By Claim 4.8,

$$
\pi_{n}^{-1} \circ \Sigma^{-1} \circ \psi_{0} \circ t^{-n}=\pi_{n}^{-1} \circ \Sigma^{-1} \circ \lambda^{-n} \circ \psi_{0}=\Psi_{n}^{-1} \circ \psi_{0} \rightarrow \operatorname{Id}_{\mathbb{H}}
$$


as $n$ tends to infinity. Taking the derivatives, we get

$$
\frac{t_{0}^{-n}}{\operatorname{Im}\left(w_{n}\right)}\left(\Sigma^{-1} \circ \psi_{0}\right)^{\prime} \circ t_{0}^{-n} \rightarrow 1
$$

uniformly on compact subsets of $\mathbb{H}$. By taking the arguments, one sees that $\Sigma^{-1} \circ \psi_{0}$ is semi-conformal at infinity; see [5], definition 2. To prove that $\Sigma^{-1} \circ \psi_{0}$ has an angular derivative at infinity, it would be enough to show that $t_{0}^{n} \operatorname{Im}\left(w_{n}\right)$ converges as $n$ tends to infinity. Notice that $\Sigma^{-1} \circ \Psi_{n}=\Phi_{n} \circ \pi_{n}$. Thus, $\left(\Phi_{n} \circ \pi_{n}\right)^{\prime}$ converges to $\left(\Sigma^{-1} \circ \psi_{0}\right)^{\prime}$ uniformly on compact subsets of $\mathbb{H}$. Evaluating at $i$ and multiplying above and below by $t_{0}^{n}$, we obtain

$$
t_{0}^{n} \operatorname{Im}\left(w_{n}\right) \prod_{j=1}^{n} \frac{\Phi^{\prime}\left(w_{j}\right)}{t_{0}} \longrightarrow\left(\Sigma^{-1} \circ \psi_{0}\right)^{\prime}(i) .
$$

So $t_{0}^{n} \operatorname{Im}\left(w_{n}\right)$ converges if and only if the infinite product converges, i.e. if and only if $\sum_{j=1}^{\infty}\left(t_{0}-\Phi^{\prime}\left(w_{j}\right)\right)$ converges.

However, consider the following example. Let $\mathbb{S}=\{x+i y:|y|<\pi / 2\}$ be the "standard strip". Let

$$
\mathcal{G}=\{x+i y: x<1,|y|<\pi\} \cup\left\{x+i y: x>1,|y|<\frac{\pi}{2}+\frac{1}{x}\right\} .
$$

Then, $\mathcal{G}-1 \subset \mathcal{G}$. By exponentiating, $\mathcal{G}$ is transformed into a region $G \subset \mathbb{C} \backslash(-\infty, 0]$ such that $e^{-1} G \subset G$ and $\mathbb{D} \backslash(-1,0] \subset G$. Hence, by adding the slit $(-1,0]$ to such a region $G$, we obtain a geometric model. Iterating translation by -1 on $\mathcal{G}$, we are left with $\mathbb{S}$. Therefore, the invariant set in this case has only one component, which is periodic of period 1. Let $\Sigma$ denote the Kœenigs map from $\mathbb{H}$ to $G \cup \mathbb{D}$. Consider $\tilde{\Sigma}(z)=\Sigma\left(i e^{z}\right)$ defined on $\mathbb{S}$. There is a curve $\gamma \subset \mathbb{S}$ starting at 0 , with $\sup \{\operatorname{Re}(z): z \in \gamma\}<+\infty$, such that $\tilde{\Sigma} \operatorname{maps} \gamma$ onto $(-1,0]$. So, $F=\log \tilde{\Sigma}$ maps $\mathbb{S} \backslash \gamma$ conformally onto $\mathcal{G}$. Moreover, $F$ does not have an angular derivative at $+\infty$; see Definition 7. of [5], because $\operatorname{Area}(\{z \in \mathcal{G} \backslash \mathbb{S}: \operatorname{Re}(z)>s\})=\infty$, for all $s>0$. This can be shown using Ahlfors's distortion theorem; see equation (15) of [5]. In particular, $F^{-1}$ restricted to $\mathbb{S}$ also doesn't have an angular derivative at $+\infty$.

Now, the map $\psi_{0}$ of $\mathbb{H}$ onto the periodic component $\{z \in \mathbb{C}: \operatorname{Re}(z)>0\}$ can be chosen to be $\psi_{0}(z)=-i z$. Thus, the map

$$
\Sigma^{-1} \circ \psi_{0}=i \exp \left(\left(F^{-1}\right)_{\mid \mathbb{S}}\left(\log \left(\psi_{0}\right)\right)\right)
$$

does not have an angular derivative at infinity.

\section{REFERENCES}

1. C. Cowen and B. MacCluer, Spectra of some composition operators, J. Functional Analysis, 125, (1994), 223-251. MR 95i:47058

2. C. Cowen and B. MacCluer, Composition operators on spaces of analytic functions, CRC Press, 1995. CMP 96:14

3. P. Duren, Theory of $H^{p}$ spaces, Academic Press, 1970. MR 42:3552

4. H. Kamowitz, The spectra of composition operators on $H^{p}$, J. Functional Analysis, 18, (1975), 132-150. MR 53:11417

5. D. Marshall, Angular derivatives and Lipschitz majorants, preprint.

6. P. Poggi-Corradini, Hardy spaces and twisted sectors for geometric models, Trans. Amer. Math. Soc., 348, (1996), 2503-2518. MR 97e:30063

7. P. Poggi-Corradini, The Hardy class of geometric models and the essential spectral radius of composition operators, J. Functional Analysis, 143, (1997), 129-156. CMP 97:06

8. C. Pommerenke, Boundary behaviour of conformal maps, Springer-Verlag, 1992. MR 95b:3008 
9. J. Shapiro, Composition operators and classical function theory, Springer-Verlag, 1993. MR 94k: 47049

10. J. Shapiro, W. Smith, D. Stegenga, Geometric models and compactness of composition operators, J. Functional Analysis, 127, (1995), 21-62. MR 95m:47051

Department of Mathematics, University of Virginia, Charlottesville, Virginia 22903

E-mail address: pp2n@virginia.edu 